\title{
A high-resolution genomic composition- based method with the ability to distinguish similar bacterial organisms
}

\author{
Yizhuang Zhou ${ }^{1,2^{*}}$ (D) Wenting Zhang ${ }^{1}$, Huixian $\mathrm{Wu}^{3,4}$, Kai Huang ${ }^{1,3,4}$ and Junfei Jin ${ }^{1,3,4^{*}}$
}

\begin{abstract}
Background: Genomic composition has been found to be species specific and is used to differentiate bacterial species. To date, almost no published composition-based approaches are able to distinguish between most closely related organisms, including intra-genus species and intra-species strains. Thus, it is necessary to develop a novel approach to address this problem.

Results: Here, we initially determine that the "tetranucleotide-derived z-value Pearson correlation coefficient" (TETRA) approach is representative of other published statistical methods. Then, we devise a novel method called "Tetranucleotide-derived Z-value Manhattan Distance" (TZMD) and compare it with the TETRA approach. Our results show that TZMD reflects the maximal genome difference, while TETRA does not in most conditions, demonstrating in theory that TZMD provides improved resolution. Additionally, our analysis of real data shows that TZMD improves species differentiation and clearly differentiates similar organisms, including similar species belonging to the same genospecies, subspecies and intraspecific strains, most of which cannot be distinguished by TETRA. Furthermore, TZMD is able to determine clonal strains with the TZMD $=0$ criterion, which intrinsically encompasses identical composition, high average nucleotide identity and high percentage of shared genomes.

Conclusions: Our extensive assessment demonstrates that TZMD has high resolution. This study is the first to propose a composition-based method for differentiating bacteria at the strain level and to demonstrate that composition is also strain specific. TZMD is a powerful tool and the first easy-to-use approach for differentiating clonal and non-clonal strains. Therefore, as the first composition-based algorithm for strain typing, TZMD will facilitate bacterial studies in the future.
\end{abstract}

Keywords: Composition, Species delineation, Metagenomic binning, Strain typing, TETRA, TZMD, Taxonomy

\section{Background}

Genomic composition refers to a set of short oligonucleotide frequencies in a genome. It can be profiled as short oligonucleotides from two to nine nucleotides [1], especially dinucleotides [2], trinucleotides [3, 4] and tetranucleotides [5]. The GC content and codon bias, which are represented by dinucleotides and trinucleotides respectively, are two examples of genomic composition. Genomic composition has been extensively studied by nearest-neighbor frequency analysis [6-8], chaos game representation $[4,9,10]$, and

\footnotetext{
* Correspondence: zhouyizhuang3@163.com; changliangzijin@163.com 'Laboratory of Hepatobiliary and Pancreatic Surgery, The Affiliated Hospital of Guilin Medical University, Guilin, Guangxi 541001, People's Republic of China

Full list of author information is available at the end of the article
}

statistical methods such as the odds ratio for dinucleotide bias [11], the Codon Adaptation Index for codon bias [12], and relative abundance measures for trinucleotides and tetranucleotides [13]. Studies show that intragenomic composition is fairly constant $[5,14,15]$, even in ameliorated horizontally transferred regions [16]. Additionally, it has been reported that closely related organisms show more similar compositions than distantly related organisms, and thus, composition has been used to infer phylogenies $[17,18]$. Furthermore, intragenomic composition is generally more homogenous than intergenomic composition [2, 9, 19-21], even between species sharing environmental pressures and interactions [22], implying that composition is species specific [14]. Thus, genomic composition is also coined genomic signature $[2,14]$. 
The greater homogeneity of intragenomic composition may possibly result from (but is not limited to) speciesspecific properties of replication and repair machineries $[2,20]$. However, whether composition is also strain specific remains unknown.

As composition is species specific, it has been widely used for species differentiation [23, 24] and metagenomic binning (classifying sequences into species-level groups) [25-31]. Additionally, composition has been used to detect foreign sequences, including laterally transferred genes [32-35], phage and viral genomes [36-39], and plasmids $[40,41]$. Although methods based on composition can distinguish most species [24], they still cannot distinguish some similar species [23, 24]. In addition, they cannot differentiate most intraspecific strains [23]. Thus, it would be very useful to develop a novel method with the ability to distinguish similar organisms, including similar species and intraspecific strains.

The ability to distinguish genomic composition increases with oligonucleotide size $[1,5,14,42]$. However, the computing cost also correspondingly increases. To balance the distinguishing ability and computing cost, tetranucleotides are thus widely used $[17,23,24,35,41,43,44]$. To our knowledge, four statistical methods have been published for tetranucleotide profiling. All of these methods use the Pearson correlation coefficient to assess composition similarity $[5,42]$. Here, we found that the tetranucleotidederived z-value Pearson correlation coefficient (TETRA) method could represent the three other statistical methods and thus could be used as the reference method for comparison when developing powerful methods. Subsequently, we proposed the tetranucleotide-derived z-value Manhat$\tan$ distance (TZMD) approach, which uses the Manhattan distance rather than the Pearson correlation coefficient to quantify composition differences, and demonstrated that genomic composition is also strain specific. Our results clearly showed that TZMD is a high-resolution method that provides slightly improved results for species differentiation and can distinguish similar organisms, including closely related species at the species level and subspecies or intraspecific strains below the species level. Most importantly, TZMD is the first genomic composition-based method to differentiate clonal and non-clonal strains. Thus, we anticipate that TZMD will be used for species differentiation or for strain typing to facilitate bacterial studies.

\section{Results}

\section{Comparison of four published statistical methods}

To date, four different statistical methods have been published to measure tetranucleotide usage biases from their expectations. The zero-order Markov method removes mononucleotide frequency biases under a random mononucleotide distribution to measure tetranucleotide frequency biases [17, 42]. The maximal-order Markov method removes component biases to calculate tetranucleotide usage biases $[17,42]$. The $\mathrm{z}$-value method, in addition to the maximal-order Markov model, takes tetranucleotide variances into account to measure tetranucleotide usage biases as z-values [5]. The relative tetranucleotide frequency method factors out all lowerorder biases to determine tetranucleotide usage biases [20]. Then, tetranucleotide usage biases calculated with all these methods are subjected to Pearson correlation coefficient calculations to measure the composition similarity between two sequences $[5,42]$.

To compare the four statistical methods, we used 1779 queries (Additional file 1: Table S1) against 264 references (Additional file 1: Table S2) comprising 1964 intraspecific and 467,692 interspecific pairs to profile the tetranucleotide usage biases and then calculated the Pearson correlation coefficients. Because the composition is species specific [2, 9, 14, 19-21], an effective method should strongly reflect this feature. Therefore, we first determined the optimal Pearson correlation coefficient cutoffs for species differentiation for these methods. The $F$-score, which was previously applied to determine the optimal sequence similarity thresholds for 40 single-copy phylogenetic marker genes [45] and 16S rRNA genes [46] for species delineation of prokaryotes, was applied to determine the optimal cutoff with the highest $F$-score for species differentiation for each method. For the statistical test, we randomly sampled 200 distinct intraspecific pairs and 50,000 distinct interspecific pairs 10 times for each sampling. We found that the optimal cutoffs for all these methods were identical at 0.99 or 1.00 (Additional file 2: Figure S1). Our paired $\mathrm{t}$-test showed that the $\mathrm{z}$-value method generated significantly higher $F$-scores than the zero-order Markov method but similar F-scores as the maximal-order Markov method and the relative tetranucleotide frequency method (Additional file 2: Figure S2). These results were further confirmed using the Rand index (Additional file 2: Figure S3 and S4). In this context, TETRA, which calculates the Pearson correlation coefficient for $\mathrm{z}$-values obtained by the z-value method, can be used as a representative method for the three other published methods. Therefore, in this study, we only compared TZMD with TETRA to show the high resolution of TZMD. In addition, our further analysis showed that the vast majority of intraspecific pairs with a Pearson correlation coefficient $>0.99$ cutoff had a Pearson correlation coefficient value of 1.00 for all four methods (Additional file 2: Figure S5), indicating that they have no ability to distinguish most intraspecific strains. Accordingly, there is a clear need for more powerful approaches.

\section{Proposal of the TZMD approach}

TETRA cannot differentiate closely related species, such as Campylobacter jejuni and C.coli [23], and most 
intraspecific strains, as shown by our above findings (Additional file 2: Figure S5). There are two possible reasons: one is that these organisms have almost no differences in composition and the other is that the resolution of TETRA is too low to distinguish these organisms, although they have different compositions. We found that some of the inability to differentiate closely related species or intraspecific strains was caused by the low resolution of TETRA. Taking the intraspecific pair Burkholderia ubonensis MSMB1189WGS and B. ubonensis RF23-BP41 and the interspecific pair B. ubonensis MSMB1189WGS and B. vietnamiensis G4 as examples, the intraspecific pair had almost identical composition yielding a TETRA value of 1.00 , as the two curves almost completely coincided (Fig. 1a). Nevertheless, we found that TETRA also yielded an undistinguishable TETRA value of 0.99 for the interspecific pair with clearly different compositions (Fig. 1b), according to the above-determined cutoff of 0.99 (Additional file 2: Figure S1 and S3). This finding demonstrated that TETRA truly had low resolution to distinguish closely-related species, which was one of the limitations of the TETRA approach.

One possible reason for the low resolution of TETRA is that the Pearson correlation coefficient cannot efficiently measure the individual $\mathrm{z}$-value difference, as demonstrated by the example shown in Fig. 1b delineated by a dotted oval. From a mathematical perspective, the Pearson correlation coefficient reflects a general trend for all $256 \mathrm{z}$-values, while the Manhattan distance efficiently reflects the z-value difference for each individual tetranucleotide (see Methods), implying that using the Manhattan distance instead of the Pearson correlation coefficient may improve the resolution to measure the composition difference. Accordingly, we proposed TZMD, a novel method using the Manhattan distance, and anticipated that it would increase the resolution for tetranucleotide usage biases.

When calculating z-values for $10-100 \%$ of the genome, we found that the tetranucleotide deviation (usage bias including over- and underrepresentation) increased with sequence size (Additional file 2: Figure S6A), which was more clearly demonstrated by using the accumulated tetranucleotide deviations (Fig. 2a). We showed that the sequence size greatly affected the TZMD (Additional file 1: Table S1), while it did not affect the TETRA. To eliminate the impact of sequence size, we normalized the $\mathrm{z}$ values by dividing by the square root of the sequence size. After normalization, differently sized sequences from the same genome expectedly yielded similar deviations (Fig. 2b and Additional file 2: Figure S6B), although $10 \%$ of the genome generated relatively different deviations due to the skewed composition for short sequences (Fig. 2b). This finding demonstrated that our method for normalization is correct. Thus, the normalized z-values can be used for TZMD calculation since they accurately reflect genomic composition. We calculated the TZMD

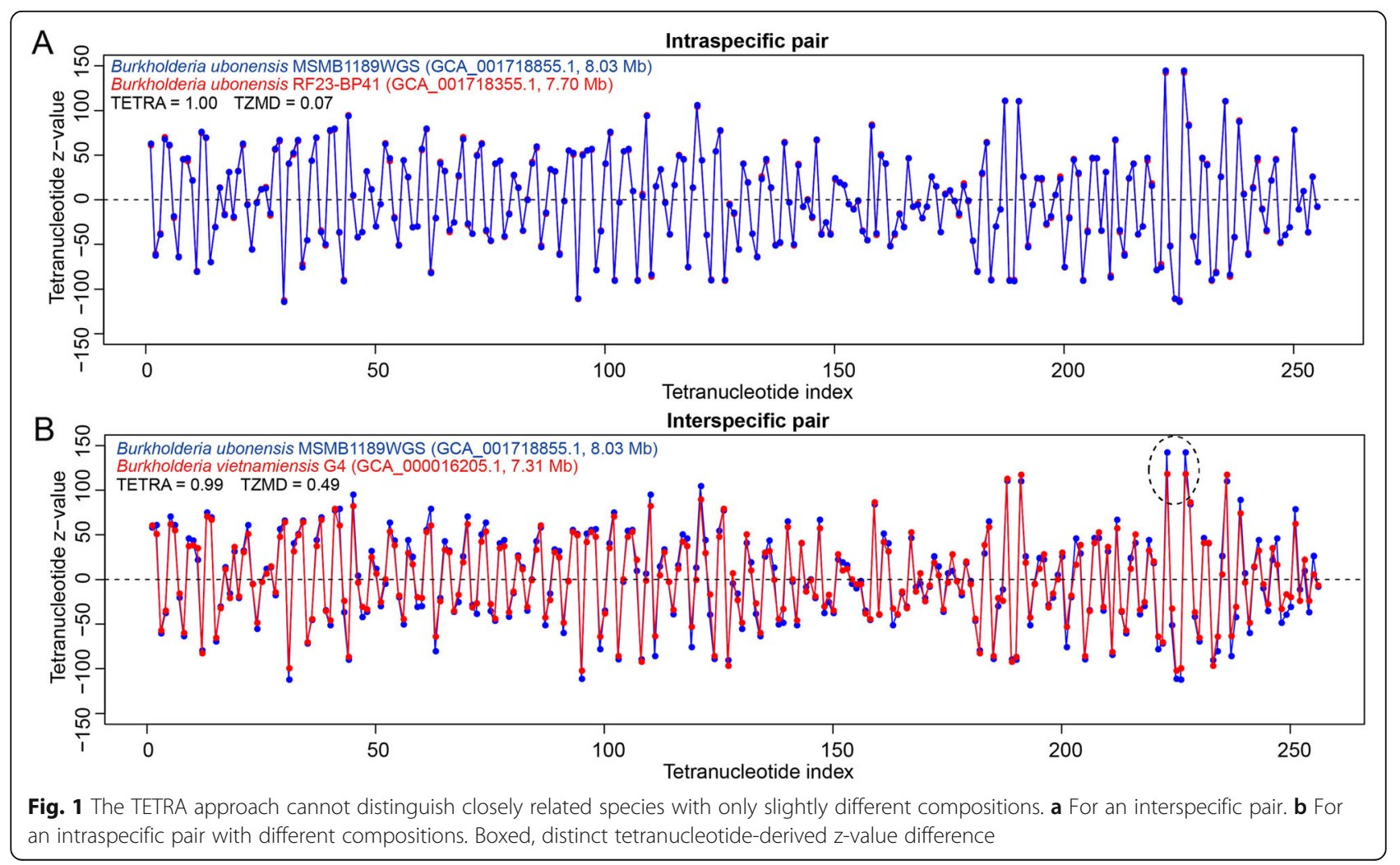



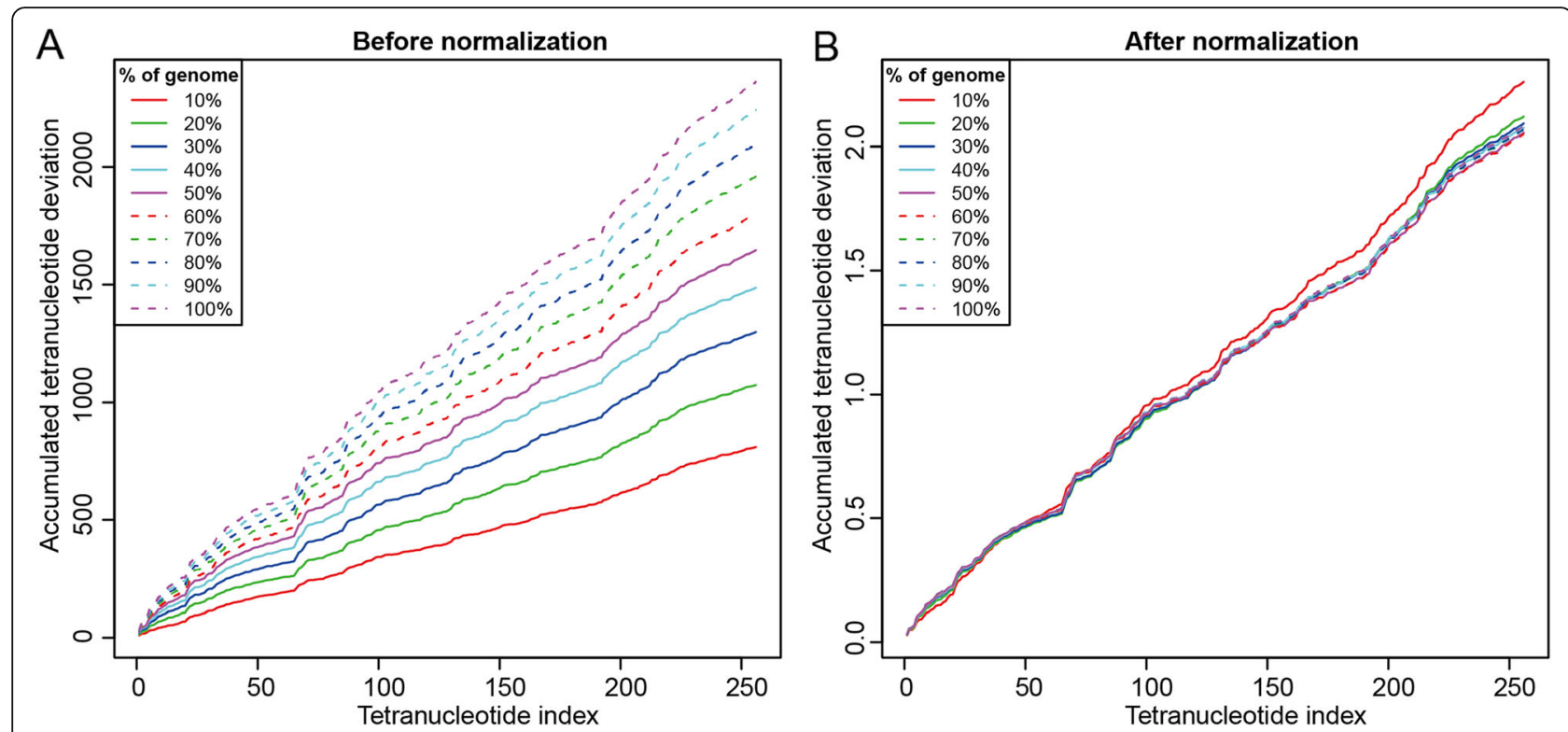

Fig. 2 Normalization of tetranucleotide-derived z-values. a Before normalization. b After normalization. Values shown here represent the accumulated tetranucleotide deviations for Buchnera aphidicola str. APS (Acyrthosiphon pisum) (GCA_000009605.1)

based on the normalized z-values of the aforementioned two pairs and found that our TZMD approach generated two distinguishable values (Fig. 1) according to the below-determined TZMD cutoff of 0.21 (see below), preliminarily showing that TZMD has a higher resolution than TETRA.

\section{Reflecting the maximal genomic difference}

Although both TETRA and TZMD quantify composition similarity/difference, our results from the aforementioned 1779 queries against 264 references showed that TZMD was only moderately correlated with TETRA in a power fashion $\left(R^{2}=0.7291\right)$, indicating that TZMD is not simply a fine-tuned version of TETRA (Fig. 3a). Accordingly, we explored whether TZMD or TETRA was more robust in reflecting genomic differences. Genomic differences between two genomes encompass two aspects: the percentage of shared genome (PSG) and average nucleotide identity (ANI) of the shared genome. For a given pair, we calculated two PSGs: one for the smaller PSG (termed PSG $_{\text {small }}$ ) and the other for the larger PSG (termed $\mathrm{PSG}_{\text {large }}$ ). In addition, we calculated a medium PSG that was an average of the two PSGs (termed $\mathrm{PSG}_{\text {mean }}$ ). In total, there were seven different measures including ANI, PSG small $_{\text {PSG }}$ mean and $\mathrm{PSG}_{\text {large }}$ for one aspect of genomic difference and $A N I^{*}{ }^{*} G_{\text {small }}$,

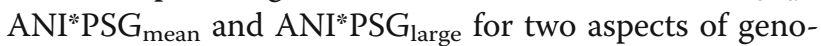
mic difference. Among them, ANI*PSG ${ }_{\text {small }}$ was the maximal difference between two genomes. Our correlation analysis showed that TZMD showed the highest $R^{2}$ values for the maximal genomic differences $\left(\mathrm{ANI}^{*} \mathrm{PSG}_{\mathrm{s}}\right.$ mall), regardless of the TZMD cutoff used (Fig. 4a). In contrast, TETRA did not give the highest $R^{2}$ values for

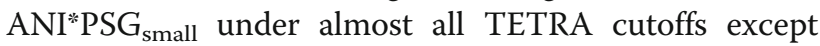
0.1 (Fig. 4b). Thus, TZMD always reflected the maximal difference, endowing it with a higher distinguishing power than TETRA. Additionally, it was noteworthy that the $R^{2}$ values for the maximal differences were only slightly higher than those for the other measures except the ANI for distantly related organisms, but relatively much higher for closely related organisms (Fig. 4a). This result indicates that the resolution difference between TZMD and TETRA arises primarily with closely related organisms, although TZMD also exhibits a slight improvement over TETRA for differentiating distantly related organisms.

Next, we explored whether TZMD was more robust in distinguishing similar organisms at or below the species level. Tracking for the 1779 queries against 264 references revealed that almost all intraspecific TETRA values were $>0.99$ with only two exceptions: one for the Borrelia hermsii strains CC1 and HS1 with an atypical TETRA of 0.97 and the other for the Borreliella burgdorferi strains CA382 and B31 with an atypical TETRA of 0.95 . Correspondingly, these exceptions had an atypical TZMD of 0.31 and 0.42 respectively (Additional file 2: Figure S7). Excluding these two pairs, all other intraspecific pairs had a maximal TZMD of 0.27 , which was used as the TZMD cutoff to evaluate the distinguishing ability of TZMD for similar organisms. We found that TZMD yielded a higher $R^{2}$ value under its cutoff of 0.27 than TETRA when its cutoff was set at 0.99 , theoretically demonstrating that TZMD is more robust in distinguishing organisms at or below the species level, 


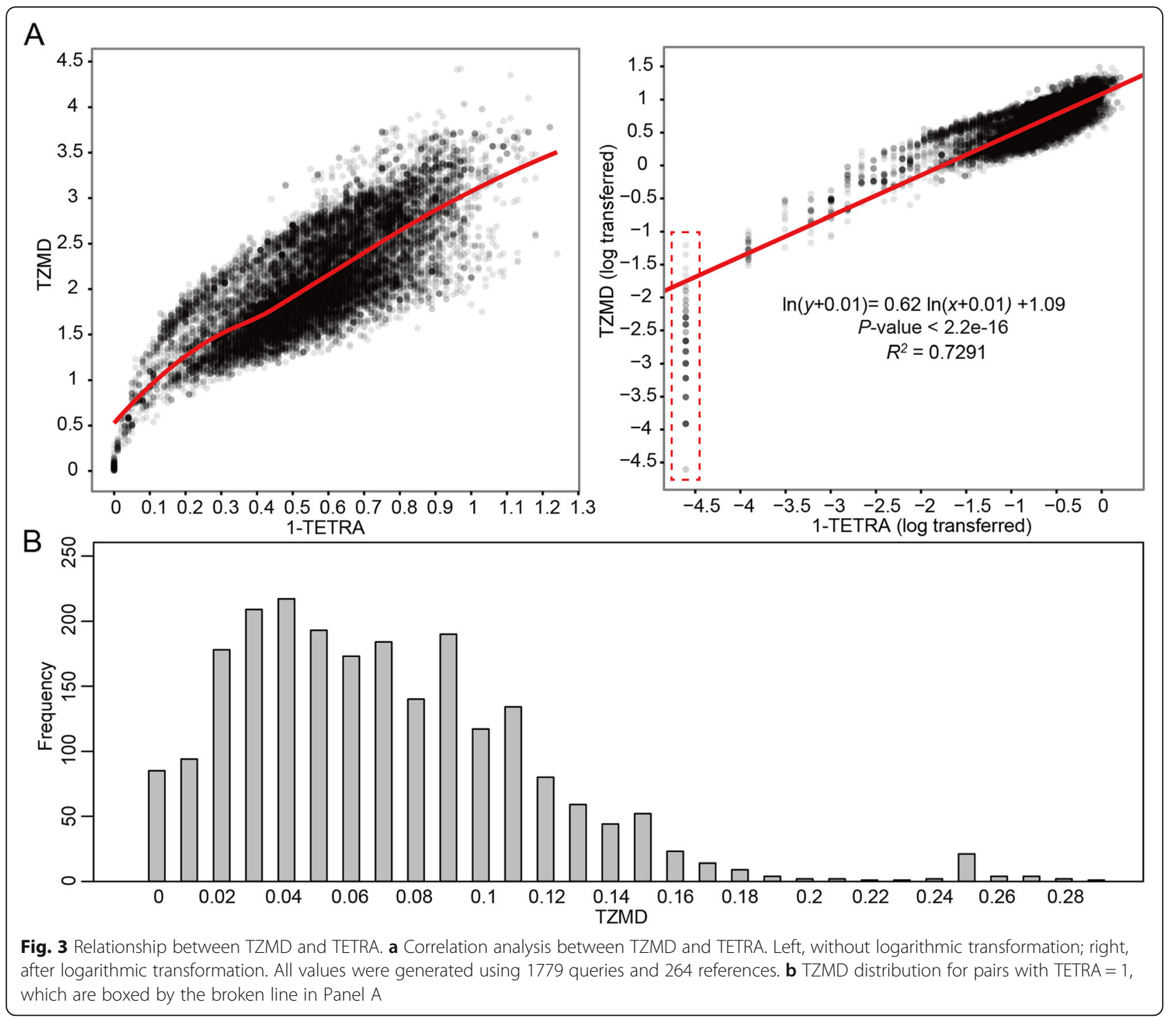

including species belonging to a single genospecies at the species level, and subspecies or intraspecific strains below the species level.

In conclusion, from the theoretical viewpoint, we demonstrated that TZMD has a higher resolution than TETRA. As an example, the pairs with a TETRA of 1.00, which were considered to have completely identical compositions by the TETRA approach, were found to show distinguishable TZMD values ranging from 0 to 0.29 (Fig. 3b), supporting that TZMD has a higher resolution than TETRA.

\section{Slight improvement in species differentiation}

Genomic composition is species specific, and intraspecific differences are generally lower than interspecific differences [2, 9, 47, 48]. Thus, TETRA has been widely applied at or above the species level, such as in metagenomic binning $[5,28]$ and species differentiation [24]. However, although TETRA performs very well in most conditions, it cannot distinguish certain closely related species, especially intra-genus species [23]. One possible reason is that TETRA incompletely quantifies genomic differences (Fig. 4b). In contrast, TZMD reflects the maximal difference between genomes. Thus, we investigated whether TZMD could improve species differentiation.

For species differentiation, we first determined the optimal species-level cutoff for TZMD. Here, we used the 1779 queries against 264 references to determine the optimal species-level cutoff. The optimal cutoff was determined to be 0.21 with both the highest $F$-score (precision $=0.8688$, recall $=0.9949) \quad$ (Fig. 5a) and the highest Rand index (Additional file 2: Figure S8A). Using the 0.21 criterion, TZMD correctly differentiated 1954 intraspecific and 467,397 interspecific pairs to achieve a 

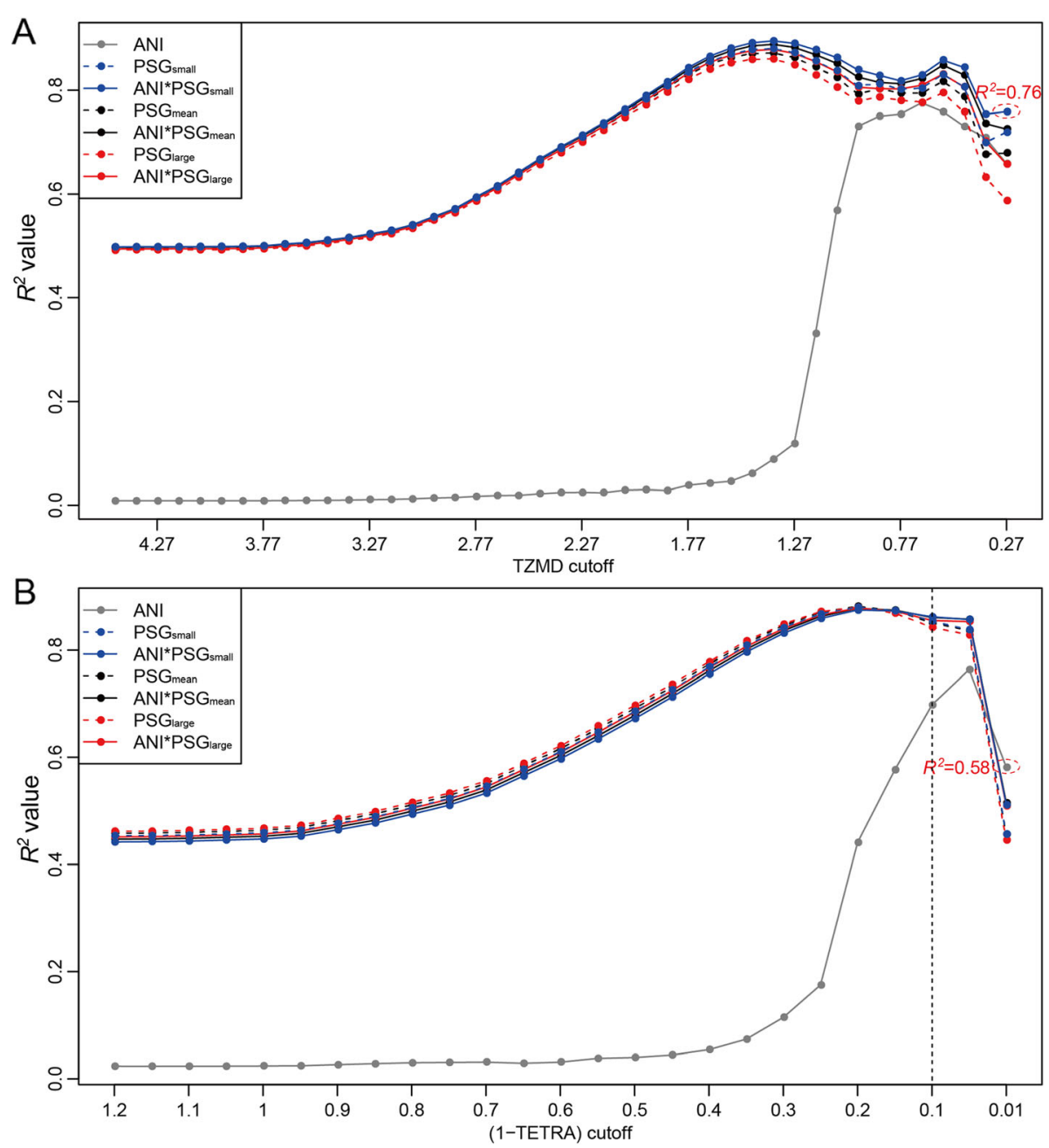

Fig. 4 TZMD reflects the maximal genome difference. a Correlation results for TZMD. b Correlation results for TETRA. A total of 1779 queries against 264 references were used. The correlation results for organisms at or below the species level are indicated by the dotted ovals. TETRA gives the highest $R^{2}$ values for measures other than the maximal difference (ANI*PSG $\mathrm{s}_{\text {small }}$ ) except when TETRA $>0.90$ (indicated by a vertical dotted line)

high Rand index of $~ 0.9994$, while TETRA correctly differentiated 1962 intraspecific and 466,776 interspecific or 1918 intraspecific and 467,371 interspecific pairs to achieve a relatively low Rand index of $\sim 0.9980$ or $\sim$ 0.9992 (Additional file 2: Figure S8B) when using the 0.99 or 1.00 criterion respectively (Additional file 2: Figure S1A and S3A). For the statistical test, we randomly sampled 200 distinct intraspecific pairs and 50,000 distinct interspecific pairs for each sampling 10 times for both methods. The results showed that the optimal cutoff for TZMD could also be determined at 0.21 (Fig. $5 \mathrm{~b}$ and Additional file 2: Figure S8C), which was in line with the above finding (Fig. 5a and Additional file 2: Figure S8A). A paired t-test showed that TZMD significantly outperformed TETRA (Fig. 5c and Additional file 2: Figure S8D). Therefore, from the perspective of species differentiation, we demonstrated that TZMD had a higher resolution than TETRA. However, compared with TETRA,
TZMD exhibited only a slight improvement in species differentiation, possibly because the maximal genomic differences (ANI*PSG small ) reflected by TZMD were only slightly superior to the $\mathrm{PSG}_{\text {large }}$ or other measures reflected by TETRA for distantly related organisms (Fig. 4a).

\section{Differentiating similar species belonging to a single genospecies}

Closely related species, such as Escherichia coli-Shigella [24] and Bacillus anthracis-Bacillus thuringiensis-Bacillus cereus [46], were defined as a single genospecies by the methods based on overall genotypic similarity, such as the ANI approach. Generally, TETRA cannot distinguish most closely related species. As an alternative, we considered whether TZMD could differentiate between these species. Brucella species were taken as an example for testing, as they were delineated as a single species by the DNA-DNA hybridization method due to their $>90 \%$ 


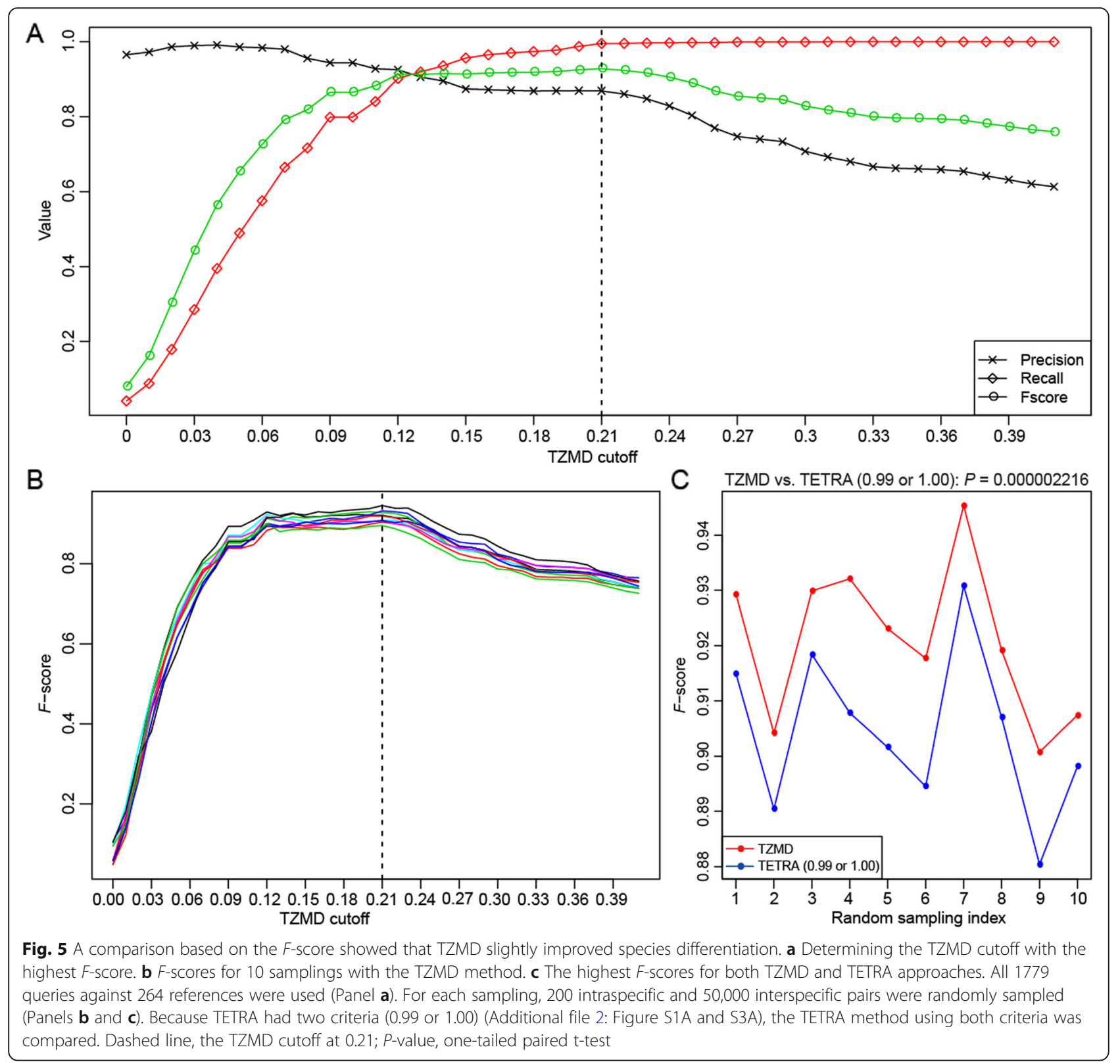

DNA-DNA hybridization values $[49,50]$ and by the ANI approach due to their $>96 \%$ ANIs [46]. We collected 53 complete genomes from the National Center for Biotechnology Information (NCBI) database (Additional file 1: Table S3) and used them to test whether TZMD could differentiate similar species belonging to a single genospecies. As expected, TETRA could not differentiate any Brucella species (Additional file 2: Figure S9). However, strikingly, TZMD clearly differentiated all Brucella species (Fig. 6). In addition, TZMD further separated B. suis biovars into three main clades (513UK, biovar 2 and other biovars including biovars 1, 3 and 4), which was consistent with the phylogenetic results based on genome-wide singlenucleotide polymorphisms (SNPs), multilocus sequencing typing and whole-genome sequence alignment [51]. Additionally, TZMD showed that B. canis might have evolved from the clade of "other biovars" of $B$. suis, in line with the findings of a previous study [52]. In addition, the highest TZMD value was 0.19 for the pair B. vulpis F60 and B. suis bv. 1 str. S2, followed by 0.18 for the pairs of B. vulpis F60 with all other strains and $<0.1$ for all other pairs (Fig. 6). Therefore, according to its optimal cutoff for species differentiation (Fig. 5a), TZMD only delineated all Brucella species as a single genospecies, in accordance with previous studies $[46,49,50]$.

Yersinia pseudotuberculosis-Yersinia pestis and Burkholderia mallei-Burkholderia pseudomallei, both of which were considered as a single genospecies by the 


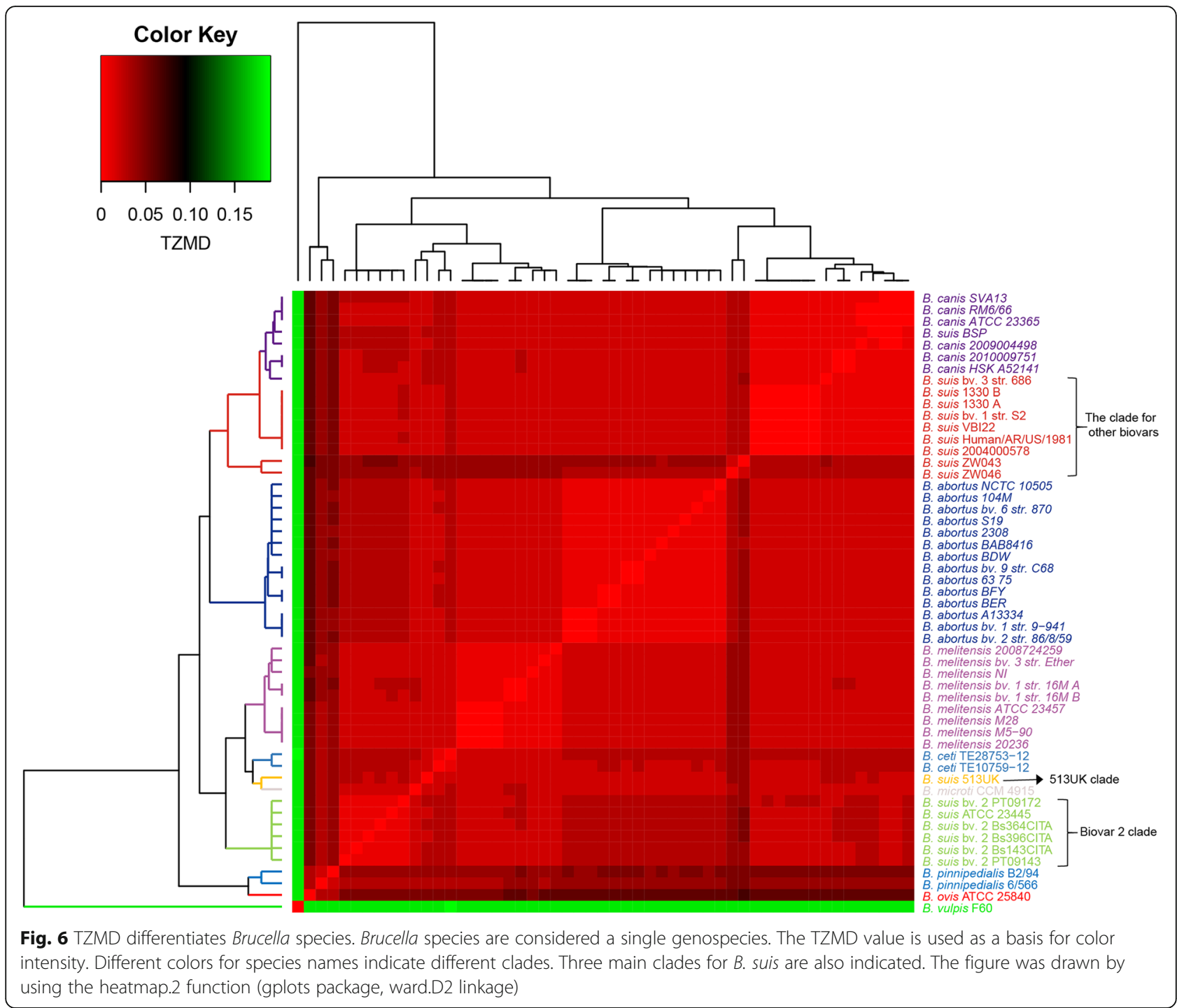

ANI approach [46], were used as two other examples. In total, 45 complete genomes of $Y$. pseudotuberculosis-Y. pestis (Additional file 1: Table S4) and 67 complete genomes of B. mallei-B. pseudomallei (Additional file 1 Table S5) were collected from the NCBI database for analysis. TETRA could not distinguish between $Y$. pestis and $Y$. pseudotuberculosis (Additional file 2: Figure S10). By contrast, TZMD clearly differentiated them (Additional file 2: Figure S11). Similar results were also obtained for B. mallei-B. pseudomallei (Additional file 2: Figure S13 and S14). In conclusion, all these findings at the species level demonstrated that TZMD has a higher resolution than TETRA.

\section{Differentiating subspecies and intraspecific strains}

Next, we tested whether TZMD could be used to differentiate subspecies and intraspecific strains. $C$. jejuni, one of the major foodborne pathogens in the world, causes enteritis or Guillain-Barre syndrome in humans [53-55].
C. jejuni has two distinct subspecies: subsp. jejuni and subsp. doylei [56]. More importantly, both subspecies have been completely sequenced. In addition, the complete genomes of numerous strains of $C$. jejuni have been determined [23]. Therefore, $C$. jejuni was selected to explore this issue. We collected all 39 C. jejuni complete genomes from the NCBI database (Additional file 1: Table S6), one for subsp. doylei and the remaining for subsp. jejuni. Our results showed that the TZMD approach clearly distinguished the two subspecies as well as intraspecific strains (Fig. 7), while the TETRA approach could not differentiate any strain or even subspecies (Additional file 2: Figure S14).

Francisella tularensis has several subspecies, including subsp. novicida, subsp. holarctica and subsp. tularensis, which were selected to further compare the abilities of TZMD and TETRA to distinguish subspecies. In total, 33 complete genomes were collected 


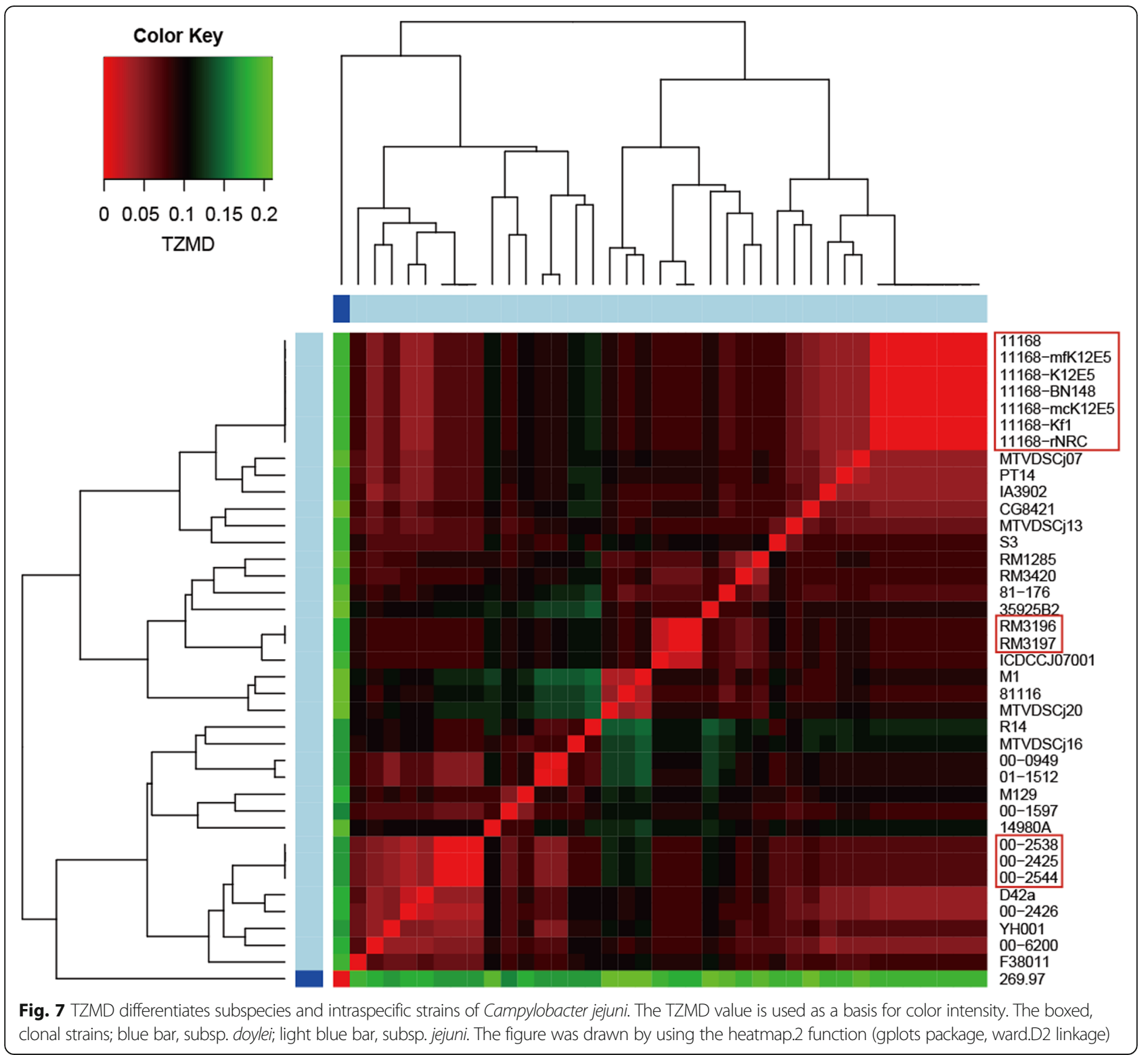

(Additional file 1: Table S7). TZMD clearly differentiated all subspecies (Additional file 2: Figure S15), while TETRA could not differentiate subsp. holarctica and subsp. novicida (Additional file 2: Figure S16).

Additional cases, including 51 complete genomes of Streptococcus pyogenes (Additional file 1: Table S8) and 53 complete genomes of Bacillus cereus (Additional file 1: Table S9), were used to further compare the abilities of TZMD and TETRA to distinguish intraspecific strains. TZMD clearly distinguished intraspecific strains of $S$. pyogenes (Additional file 2: Figure S17), while TETRA could not differentiate any strain of $S$. pyogenes (Additional file 2: Figure S18). Similar results were also found for B. cereus (Additional file 2: Figure S19 and S20).
In addition, we found that the TZMD approach further distinguished clonal and non-clonal strains for clonal strains with a TZMD value of 0 and non-clonal strains with TZMD $>0$ (Fig. 7 and Additional file 2: Figure S17 and S19). Summarizing all pairs with a TZMD value of 0 from the 1779 queries against 264 references showed that this standard included substantially more information. This standard intrinsically ensured that clonal strains had considerably high ANIs (95.85-100\%) (Fig. 8a), as well as considerably high PSGs (almost 100\%, regardless of the PSG metric used) (Fig. 8b, c and d). Therefore, this standard substantially ensured that at least three aspects (composition, ANI and PSG) were extremely similar, showing 

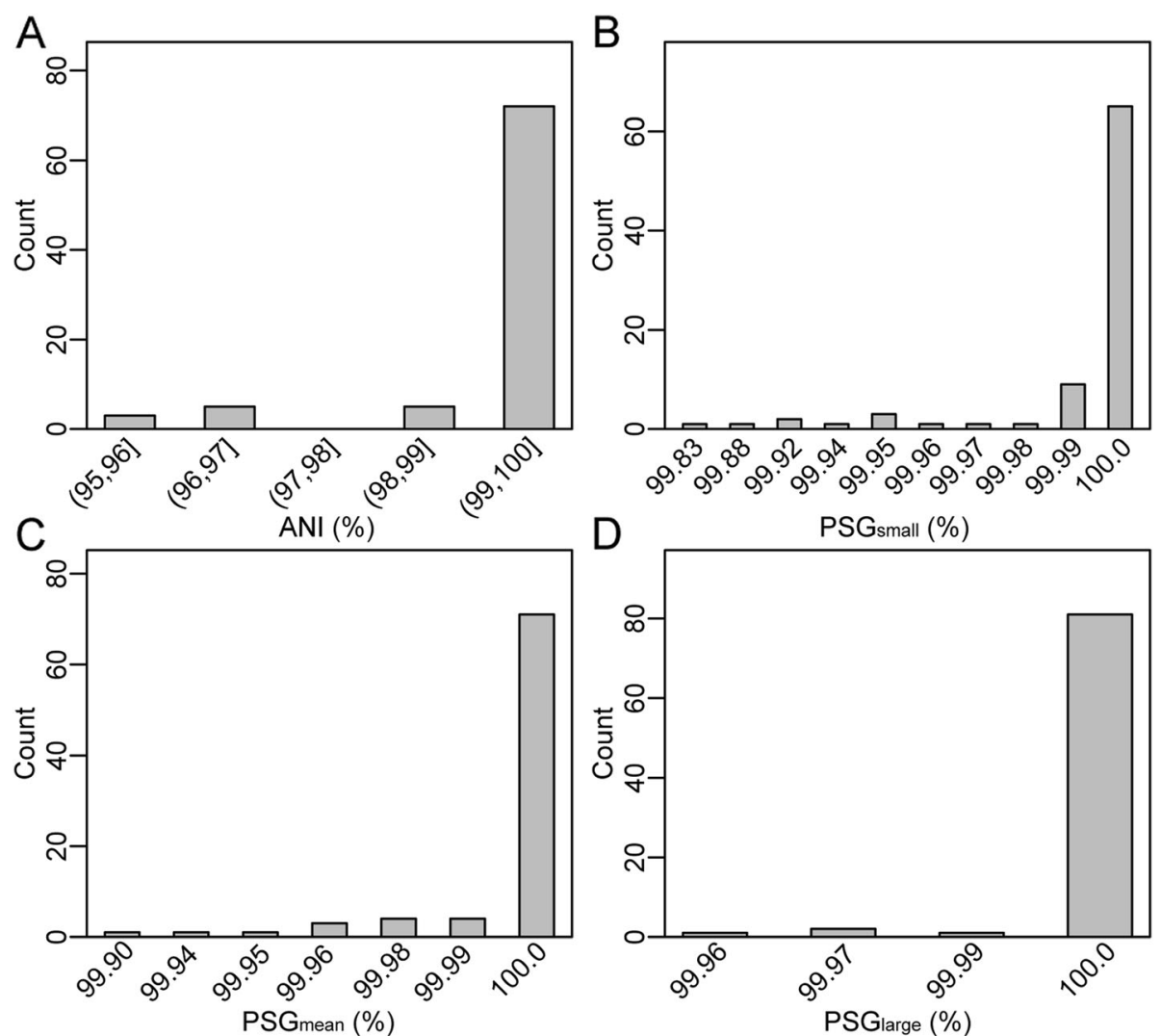

Fig. $8 \mathrm{TZMD}=0$ additionally includes high $A N I$ and high PSG. a) ANI distribution. b PSG small distribution. $\mathbf{c} P S G_{\text {mean }}$ distribution. d PSG large distribution. All values shown here are summarized from pairs with TZMD $=0$ from the 1779 queries against 264 references

that TZMD is powerful for detecting clonal strains with high confidence. All findings at the subspecies and strain levels demonstrated that TZMD has a higher resolution than TETRA.

\section{Discussion}

\section{Demonstration of high resolution}

In this study, we present TZMD, a high-resolution method for distinguishing genomic composition. We determined that TETRA was able to represent all other published statistical methods to profile tetranucleotide usage biases. Therefore, we only needed to compare our TZMD method with TETRA. We demonstrated the high resolution of TZMD by comparing it with the TETRA method from two viewpoints. First, from the theoretical point of view, we principally demonstrated that TZMD reflected the maximal difference between two genomes, endowing it with high resolution. Second, from the practical point of view, we assessed its high resolution for real data from four aspects, including species differentiation at or above the species level, differentiation of similar species belonging to a single genospecies at species level, subspecies below the species level and intraspecific strains below the species level.
TZMD exhibited a slight improvement in species differentiation, possibly because the maximal genome differences reflected by TZMD are only slightly superior to the $\mathrm{PSG}_{\text {large }}$ or other measures reflected by TETRA for distantly related organisms (Fig. 4a). However, maximal genome differences reflected by TZMD are considerably superior to the $\mathrm{PSG}_{\text {large }}$ or other measures reflected by TETRA for closely-related organisms (Fig. 4a), which is why TZMD can differentiate species belonging to a single genospecies, subspecies and intraspecific strains, whereas TETRA is almost unable to differentiate them. Taken together, these findings demonstrated that TZMD has a high resolution.

\section{Strain-specific feature of composition}

The studies for genomic composition began in the early 1960s [6]. Since then, almost all related studies have focused on the development of statistical methods and extensive demonstration and wide application of the species-specific nature of composition. In addition to the species-specific nature of composition [2, 9, 14, 19-21], our results showed that composition is also strain specific (Fig. 7). To our knowledge, this is the first study to 
demonstrate that genomic composition is strain specific. The strain-specific nature of composition is possibly due to (but not limited to) various factors. First, bacteria are evolving, which may result in SNPs, insertions or deletions. Second, some genes are acquired through horizontal gene transfer [57]. For example, genomic islands, which are horizontally transferred, carry biased composition from their core genomes [58]. Third, some genes are lost through genomic reduction [59]. Fourth, some plasmids are conjugative $[60,61]$, which may cause varied composition for their recipient genomes [41].

\section{Strain typing}

Almost all previously developed methods can distinguish only genomic composition at or above the species level, leaving a long-standing need to develop methods capable of strain-level differentiation. As far as we know, TZMD is the first genomic composition-based algorithm with the ability to differentiate similar species, subspecies and intraspecific strains and thus can be used for strain typing. Similar species, in a sense, are special intraspecific strains belonging to a single genospecies, as their TZMD values are $<0.21$ (Fig. 6). Composition Vector Tree (CVTree) can also distinguish intraspecific strains for strain typing [62]. However, CVTree uses the proteomic composition (oligopeptide) rather than the genomic composition (oligonucleotide). Accordingly, TZMD is more convenient than CVTree, as TZMD directly uses genomes, while CVTree uses proteomes requiring prediction and translation of proteincoding genes from their genomes. In addition, CVTree requires 12,500 times the computational burden of TZMD, as the composition vector of dimension for CVTree is $20^{5}$ [62], while that for TZMD is only $4^{4}$. However, our results from the present study show that TZMD is sufficiently robust to distinguish closely related species, subspecies or strains without increasing the computational burden.

Furthermore, TZMD is a powerful standard to distinguish clonal and non-clonal strains. Traditional methods are mainly based on SNPs. For example, multilocus sequence typing uses SNPs from several housekeeping genes [63]. Compared with these traditional SNP-based methods, TZMD has at least four main advantages. First, SNP-based methods only consider the information of nucleotide identity, while the TZMD standard considers at least three aspects, including ANI, PSG and composition. Second, the TZMD standard is based on genome-wide information, outperforming traditional methods based only on single or several housekeeping genes. Third, TZMD is an easy-to-use method because its standard is set at 0 , while it is difficult to set the exact SNP cutoffs for strain typing for traditional SNP-based methods. For methods using genome-wide information, the SNP cutoffs are challenging to set as the SNP cutoffs are species specific, requiring large-scale sequencing to determine the cutoffs for each species; for methods using single or multiple genes, the SNP cutoffs are also challenging to set even for the same species, as the thresholds may be different for different genes because they may be under different evolutionary stresses. Fourth, TZMD is an alignment-free method that may run faster than some traditional methods, especially genome-wide approaches, and thus reduces computing cost [24]. Taken together, these factors showed that TZMD is a powerful tool and the first easy-to-use approach for differentiating clonal and non-clonal strains.

\section{Impact of genome incompleteness}

Incomplete genomes contain only part of their genomes and thus may carry a skewed composition to yield different TZMD and TETRA values related to their full genomes (Additional file 1: Table S1). Even genomes with 90\% completeness may yield slightly different TZMD values (Additional file 1: Table S1), whereas most closely related organisms, including similar species belonging to a single genospecies, subspecies and intra-species strains, have only slightly different compositions (Figs. 6 and 7). Thus, using incomplete genomes may result in incorrect conclusions for differentiating these closely related organisms, which is why we only used complete genomes in this study. Therefore, the differentiation of closely related organisms requires complete genomes.

In contrast, genomic incompleteness has less of an impact on species differentiation. Nevertheless, genomic incompleteness may still affect the results. Although species differentiation using the $<0.21$ criterion for TZMD and the $>0.99$ or 1.00 criterion for TETRA is tolerant of some incompleteness, it is still affected by genomic completeness (Additional file 2: Figure S21). However, TZMD is more susceptible to genome incompleteness than TETRA. Thus, TZMD may be inferior to TETRA when analysing incomplete genomes, although TZMD slightly improves species differentiation when all tested genomes are complete (Fig. 5). However, both TZMD and TETRA are affected by genomic incompleteness, requiring the development of novel approaches.

\section{Metagenomic binning}

Composition has been widely used for metagenomic binning [25-31], as it was species specific $[2,9,19-21]$. TETRA has been used for binning [5, 28, 64], and the Euclidean distance has also been used for binning $[26,65]$. As the Manhattan distance has been reported to outperform the Euclidean distance [66], we herein only compared TZMD with TETRA. Our testing showed that TZMD performed worse than TETRA when fragment size was $<80 \mathrm{~kb}$ (Additional file 2: Figure S22), possibly because TZMD is more greatly affected by genome incompleteness than TETRA (Additional file 2: Figure S21), but it 
outperformed TETRA when fragment size was $>80$ $\mathrm{kb}$, possibly because TZMD performs slightly better in species differentiation. However, the difference of binning performance between them is very slight. Thus, either approach can be selected for binning.

\section{Conclusions}

Here, we develop a novel approach termed TZMD for genomic composition and extensively demonstrate that TZMD is a high-resolution method. Although traditional approaches such as TETRA are effective in distinguishing most species and thus can be successfully applied for species differentiation, TZMD slightly improves these applications. Most importantly, TZMD exclusively extends the application of composition from the species level to the strain level, endowing it with the ability to differentiate species belonging to a single species, subspecies and intraspecific strains. Furthermore, TZMD is a powerful tool and the first easy-to-use approach for differentiating clonal and non-clonal strains. Additionally, this study is the first to show that composition is strain specific. Therefore, we hope that TZMD will be used alone or in combination with TETRA to facilitate bacterial studies in the future.

\section{Methods}

\section{Calculation of PSG and ANI}

Genomes were aligned using the NUCmer tool (version 3.23) [67], with default parameters except for "--maxmatch". Then, we used the ".delta" file to calculate PSG and ANI. Stretches, such as paralogs and other repeats, overlapped in the alignment, which would introduce biases in PSG. Thus, the PSG for either genome of a pair was calculated as follows:

$$
P S G=\frac{\sum \text { Aligned positions }}{L}
$$

where the numerator is the summed aligned positions (in terms of base pairs) and the denominator is the genomic size (also in terms of base pairs). Thus, we obtained two PSGs for a given pair: the smaller one termed PSG $_{\text {small }}$ and the larger one termed PSG $_{\text {large. }}$ Additionally, an average PSG was devised and calculated as follows:

$$
P S G_{\text {mean }}=\frac{A P 1+A P 2}{L 1+L 2}
$$

where $A P 1$ and $L 1$ are the summed aligned positions and genomic size for the first genome respectively, and $A P 2$ and $L 2$ are for the second genome.

\section{Genome selection}

All prokaryotic genome sequences listed at http://www. ncbi.nlm.nih.gov/genome/ were downloaded (on 20 January 2017). Then, we selected complete genomes with only one chromosome. After this step, 5819 genomes remained. To determine the TZMD cutoff at the species level, we selected 2043 genomes with unambiguous species relationships (> 96\% ANI [24] and > 70\% of the shared gene content [68]). These genomes were separated into 1779 query genomes (Additional file 1: Table S1) and 264 reference genomes (Additional file 1: Table S2), comprising 1964 intra- and 467,692 inter-species pairs.

\section{Statistics of observed oligonucleotide frequencies}

In all cases, due to strand compositional asymmetry in certain bacterial genomes [69], all tested genomes were concatenated with their inverted complements and then processed through discarding ambiguous nucleotides (that is, not $\mathrm{A}, \mathrm{T}, \mathrm{C}$, or $\mathrm{G}$ ). The resulting sequences were compiled by moving a single base per step in the $5^{\prime}$ to $3^{\prime}$ direction with a length of $n$ nucleotides ( $n$ is $4,3,2$ or 1 ).

Four statistical methods for tetranucleotide usage biases We denote the observed frequency of an oligonucleotide as $F^{*}$.) (* indicates the oligonucleotide frequency computed from the genome extended with its reverse complementary sequence). For example, the observed frequency of the tetranucleotide $\mathrm{XYZW}$ and its component mononucleotide $\mathrm{X}$ were denoted as $F^{*}(X Y Z W)$ and $F^{*}(X)$ respectively. Then, the tetranucleotide usage biases assessed by the zero-order Markov method were written as follows [42]:

$$
\frac{F^{*}(X Y Z W)}{F^{*}(X) F^{*}(Y) F^{*}(Z) F^{*}(W)}
$$

The method of maximal-order Markov model can be calculated similarly with the following formula [42]:

$$
\frac{F^{*}(X Y Z W) F^{*}(Y Z)}{F^{*}(X Y Z) F^{*}(Y Z W)}
$$

The method of relative tetranucleotide frequency can be calculated as follows [20]:

$$
\frac{F^{*}(X Y Z W) F^{*}(X Y) F^{*}(X N Z) F^{*}\left(X N_{1} N_{2} W\right) F^{*}(Y Z) F^{*}(Y N W) F^{*}(Z W)}{F^{*}(X Y Z) F^{*}(X Y N W) F^{*}(X N Z W) F^{*}(Y Z W) F^{*}(X) F^{*}(Y) F^{*}(Z) F^{*}(W)}
$$

where $N$ is any nucleotide.

The tetranucleotide usage biases can also be measured as z-value $Z *(X Y Z W)$ by the z-value method and can be calculated as follows [5]:

$$
Z^{*}(X Y Z W)=\frac{F^{*}(X Y Z W)-E^{*}(X Y Z W)}{\sqrt{\operatorname{var}\left(F^{*}(X Y Z W)\right)}}
$$

where $E^{*}(X Y Z W)$ can be calculated using a maximalorder Markov model as follows [70]: 


$$
E^{*}(X Y Z W)=\frac{F^{*}(X Y Z) F^{*}(Y Z W)}{F^{*}(Y Z)}
$$

$\operatorname{var}\left(F^{*}(X Y Z W)\right.$ can be calculated as follows [5]:

$$
\operatorname{var}\left(F^{*}(X Y Z W)\right)=\frac{E^{*}(X Y Z W)\left[F^{*}(Y Z)-F^{*}(X Y Z)\right]\left[F^{*}(Y Z)-F^{*}(Y Z W)\right]}{F^{*}(Y Z)^{2}}
$$

Then, tetranucleotide usage biases calculated from each statistical method were subjected to calculation of the Pearson correlation coefficient to measure the composition similarity between two genomes.

\section{TZMD calculation}

The resulting sequences were subjected to calculation of tetranucleotide-derived $\mathrm{z}$-values and TETRA values according to the method published by Teeling et al. [5]. If we denote the z-value of the $k$ th tetranucleotide from the sequence $s$ as $Z_{s, k}$ and the corresponding normalized $\mathrm{z}$-value as $N Z_{s, k}$, then the zvalue can be normalized as follows:

$$
N Z_{s, k}=Z_{s, k} / \sqrt{l}
$$

where $l$ is the total length of the sequence extended with its reverse complement.

Subsequently, TZMD was calculated to measure the compositional difference between two sequences $s_{1}$ and $s_{2}$ as follows:

$$
T Z M D=\sum_{k=1}^{256}\left|N Z_{s_{1}, k}-N Z_{s_{2}, k}\right|
$$

\section{Determination of the optimal cutoffs for species differentiation}

Precision-recall and F-score were applied successfully to determine the optimal sequence similarity thresholds for 40 single-copy phylogenetic marker genes [45] and 16S rRNA genes [46] for species delineation of prokaryotes. Similarly, we used this strategy to determine the optimal TZMD cutoff for species differentiation. Briefly, all pairwise TZMD values were assigned into four categories given a threshold of $Y$ TZMD (0-0.42 at 0.01 intervals): true positives (TP) for intraspecific pairs (>96\% ANI [24] and $>70 \%$ of the shared gene content [68]) with TZMD $<Y$; false negatives (FN) for intraspecific pairs with TZMD $>Y$; false positives (FP) for interspecific pairs with TZMD $<Y$ and true negatives (TN) for interspecific pairs with TZMD $>Y$. The optimal threshold was obtained by maximizing the sensitivity (recall) as well as the precision to achieve the highest $F$-score, which is a harmonic mean of precision and recall. The $F$-score was calculated as follows:

$$
F \text {-score }=2(\text { precision } \times \text { recall }) /(\text { precision }+ \text { recall })
$$

where precision and recall were calculated as follows:

$$
\begin{aligned}
& \text { precision }=\mathrm{TP} /(\mathrm{TP}+\mathrm{FP}) \\
& \text { recall }=\mathrm{TP} /(\mathrm{TP}+\mathrm{FN})
\end{aligned}
$$

In addition, the Rand index, which is simply the number of correct assigned pairs $(\mathrm{TP}+\mathrm{TN})$ divided by the total number of tested pairs $(\mathrm{TP}+\mathrm{FN}+\mathrm{FP}+\mathrm{TN})$, was used to select the optimal threshold with the highest Rand index.

The optimal species-level thresholds for the above four statistical methods were also determined with the highest $F$-scores and Rand indexes.

\section{Supplementary information}

Supplementary information accompanies this paper at https://doi.org/10 1186/s12864-019-6119-x.

Additional file 1: Table S1. All 1,779 complete query genomes. Table S2. All 264 complete reference genomes. Table S3. A list of complete Brucella genomes used in this study. Table S4. A list of $Y$. pestis and $Y$. pseudotuberculosis genomes used in this study. Table S5. A list of B. mallei and B. pseudomallei genomes used in this study. Table S6. Campylobacter jejuni genomes used in this study. Table S7. Francisella tularensis genomes used in this study. Table S8. Streptococcus pyogenes genomes used in this study. Table S9. Bacillus cereus genomes used in this study.

Additional file 2: Figure S1. Determination of the optimal species-leve cutoffs for the four published methods based on F-score. Figure S2. Fscores for 10 samplings for each method. Figure S3. Determination of the species-level cutoffs for the four published methods based on Rand index. Figure S4. Rand indexes for 10 samplings for each method. Figure S5. Instraspecific Pearson correlation coefficient distribution for each method. Figure S6. Normalization of tetranucleotide-derived zvalues. Figure S7. Histrogram showing the TZMD distributions for both intraspecific and interspecific pairs. Figure S8. Comparison base on Rand index showed TZMD slightly improved species differentiation. Figure S9. TETRA cannot differentiate Brucella species belonging to one single genospecies. Figure S10. TETRA cannot differentiate Y. pseudotuberculosis and $Y$. pestis belonging to one single genospecies. Figure S11. TZMD differentiates $Y$. pestis and $Y$. pseudotuberculosis belonging to one single genospecies. Figure S12. TETRA cannot differentiate B. mallei and B. pseudomallei belonging to one single genospecies. Figure S13. TZMD differentiates $B$. mallei and B. pesudomallei belonging to one single genospecies. Figure S14. TETRA cannot differentiates sub species and intraspecific strains of Campylobacter jejuni. Figure S15. TZMD differentiates all subspecies of Franscisella tularensis. Figure S16. TETRA cannot differentiate two subspecies of Francisella tularensis. Figure S17. TZMD distinguishes intraspecific strains of Streptococcus pyogenes. Figure S18. TETRA cannot differentiate intraspecific strains of Streptococcus pyogenes. Figure S19. TZMD distinguishes intraspecific strains of Bacillus cereus. Figure S20. TETRA cannot differentiate intraspecific strains of Bacillus cereus. Figure S21. Impact of genomic completeness on TZMD and TETRA for species differentiation. Figure S22. Binning performance of TZMD and TETRA. Table S1. TZMD and TETRA values for differently-sized genomes.

\section{Abbreviations}

ANI: Average nucleotide identity; CVTree: Composition Vector Tree; FN: False negatives; FP: False positives; NCBI: National Center for Biotechnology Information; PSG: Percentage of shared genome; PSG large: The larger PSG; PSG mean: Average of the two PSGs; PSG small: The smaller PSG; SNP: Singlenucleotide polymorphism; TETRA: Tetranucleotide-derived z-value Pearson 
correlation coefficient; TN: True negatives; TP: True positives; TZMD: Tetranucleotide-derived Z-value Manhattan Distance

\section{Acknowledgements}

We thank Dr. lain C. Bruce (Guest Professor, Peking University) for reading the manuscript.

\section{Authors' contributions}

$Y Z$ performed the main analysis, wrote the manuscript and obtained the funding. WZ, HW, and $\mathrm{KH}$ assisted in performing the analysis and discussing the results. JJ designed this study, obtained the funding and revised the manuscript. All authors read and approved the final manuscript.

\section{Funding}

This work was supported in part by Guangxi Research Foundation for Science \&Technology Base and Talent Special (No. AD19110054) for Yizhuang Zhou and the Natural Science Foundation of Guangxi (No. 2015GXNSFEA139003), the Lijiang Scholar Award in Guilin, the High Level of Innovation Team and Outstanding Scholars Program in Colleges and Universities in Guangxi, and the Hundred Talents Program "the Introduction of Overseas High-Level Talents in Colleges and Universities in Guangxi" for Junfei Jin.

\section{Availability of data and materials}

All data generated or analyzed during this study are included supplementary information files (see tables in Additional file 1). Additionally, the TZMD approach and all tested data are available at https://github.com/Yizhuangzhou/TZMD.

\section{Ethics approval and consent to participate}

Not applicable.

\section{Consent for publication}

Not applicable.

\section{Competing interests}

The authors declare that they have no competing interests.

\section{Author details}

'Laboratory of Hepatobiliary and Pancreatic Surgery, The Affiliated Hospital of Guilin Medical University, Guilin, Guangxi 541001, People's Republic of China. ${ }^{2}$ Peking-Tsinghua Center for Life Science, Academy for Advanced Interdisciplinary Studies, Peking University, Beijing 100871, People's Republic of China. ${ }^{3}$ China-USA Lipids in Health and Disease Research Center, Guilin Medical University, Guilin, Guangxi 541001, People's Republic of China. ${ }^{4}$ Guangxi Key Laboratory of Molecular Medicine in Liver Injury and Repair, Guilin Medical University, Guilin, Guangxi 541001, People's Republic of China.

Received: 26 June 2019 Accepted: 20 September 2019

Published online: 21 October 2019

\section{References}

1. Sandberg R, Winberg G, Branden C, Kaske A, Ernberg I, Coster J. Capturing whole-genome characteristics in short sequences using a Naïve Bayesian classifier. Genome Res. 2001:11(8):1404-9.

2. Kariin S, Burge CB. Dinucleotide relative abundance extremes: a genomic signature. Trends Genet. 1995;11(7):283-90.

3. Karlin S, Burge C, Campbell AM. Statistical analyses of counts and distributions of restriction sites in DNA sequences. Nucleic Acids Res. 1992; 20(6):1363-70

4. Goldman N. Nucleotide, dinucleotide and trinucleotide frequencies explain patterns observed in chaos game representations of DNA sequences. Nucleic Acids Res. 1993;21(10):2487-91.

5. Teeling H, Meyerdierks A, Bauer M, Amann R, Glockner FO. Application of tetranucleotide frequencies for the assignment of genomic fragments. Environ Microbiol. 2004:6(9):938-47.

6. Josse J, Kaiser AD, Kornberg A. Enzymatic synthesis of deoxyribonucleic acid. VIII. Frequencies of nearest Neighbor Base sequences in deoxyribonucleic acid. J Biol Chem. 1961;236(3):864-75.

7. Russell GJ, Walker PMB, Elton RA, Subak-Sharpe JH. Doublet frequency analysis of fractionated vertebrate nuclear DNA. J Mol Biol. 1976;108(1):1-20.
8. Russell GJ, Subaksharpe JH. Similarity of the general designs of protochordates and invertebrates. Nature. 1977:266(5602):533-6.

9. Deschavanne PJ, Giron A, Vilain J, Fagot G, Fertil B. Genomic signature: characterization and classification of species assessed by chaos game representation of sequences. Mol Biol Evol. 1999;16(10):1391-9.

10. Wang $Y$, Hill KA, Singh $S M$, Kari $L$. The spectrum of genomic signatures: from dinucleotides to chaos game representation. Gene. 2005;346:173-85

11. Karlin S, Campbell AM, Mrazek J. Comparative DNA analysis across diverse genomes. Annu Rev Genet. 2003;32(1):185-225.

12. Sharp PM, Li W. The codon adaptation index-a measure of directional synonymous codon usage bias, and its potential applications. Nucleic Acids Res. 1987;15(3):1281-95.

13. Karlin S, Cardon LR. Computational DNA sequence analysis. Annu Rev Microbiol. 1994;48(1):619-54

14. Abe T, Kanaya S, Kinouchi M, Ichiba $Y$, Kozuki T, Ikemura T. Informatics for unveiling hidden genome signatures. Genome Res. 2003;13(4):693-702.

15. Bohlin J, Skjerve E, Ussery DW. Investigations of Oligonucleotide Usage Variance Within and Between Prokaryotes. PLoS Comput Biol. 2008;4(4):e1000057.

16. Lawrence JG, Ochman $\mathrm{H}$. Amelioration of bacterial genomes: rates of change and exchange. J Mol Evol. 1997;44(4):383-97.

17. Pride DT, Meinersmann RJ, Wassenaar TM, Blaser MJ. Evolutionary implications of microbial genome tetranucleotide frequency biases. Genome Res. 2003;13(2):145-58.

18. Mrazek J. Phylogenetic signals in DNA composition: limitations and prospects. Mol Biol Evol. 2009;26(5):1163-9.

19. Karlin S, Ladunga I, Blaisdell BE. Heterogeneity of genomes: measures and values. Proc Natl Acad Sci U S A. 1994;91(26):12837-41.

20. Karlin S, Mrazek J, Campbell AM. Compositional biases of bacterial genomes and evolutionary implications. J Bacteriol. 1997;179(12):3899-913.

21. Nakashima H, Ota M, Nishikawa K, Ooi T. Genes from nine genomes are separated into their organisms in the dinucleotide composition space. DNA Res. 1998:5(5):251-9.

22. Dick GJ, Andersson AF, Baker BJ, Simmons SL, Thomas BC, Yelton AP, Banfield JF. Community-wide analysis of microbial genome sequence signatures. Genome Biol. 2009;10(8):R85.

23. Zhou Y, Bu L, Guo M, Zhou C, Wang Y, Chen L, Liu J. Comprehensive genomic characterization of campylobacter genus reveals some underlying mechanisms for its genomic diversification. PLoS One. 2013:8(8):e70241.

24. Richter M, Rossello-Mora R. Shifting the genomic gold standard for the prokaryotic species definition. Proc Natl Acad Sci U S A. 2009:106(45):19126-31.

25. Alneberg J, Bjarnason BS, de Bruijn I, Schirmer M, Quick J, ljaz UZ, Lahti L, Loman NJ, Andersson AF, Quince C. Binning metagenomic contigs by coverage and composition. Nat Methods. 2014;11(11):1144-6.

26. Kang DD, Froula J, Egan R, Wang Z. MetaBAT, an efficient tool for accurately reconstructing single genomes from complex microbial communities. PeerJ. 2015:3:e1165.

27. McHardy AC, Martin HG, Tsirigos A, Hugenholtz P, Rigoutsos I. Accurate phylogenetic classification of variable-length DNA fragments. Nat Methods. 2007:4(1):63-72.

28. Teeling H, Waldmann J, Lombardot T, Bauer M, Glockner FO. TETRA: a webservice and a stand-alone program for the analysis and comparison of tetranucleotide usage patterns in DNA sequences. BMC Bioinformatics. 2004;5:163.

29. Wu YW, Tang YH, Tringe SG, Simmons BA, Singer SW. MaxBin: an automated binning method to recover individual genomes from metagenomes using an expectation-maximization algorithm. Microbiome. 2014;2:26.

30. Wu YW, Simmons BA, Singer SW. MaxBin 2.0: an automated binning algorithm to recover genomes from multiple metagenomic datasets. Bioinformatics. 2016;32(4):605-7.

31. Leung HC, Yiu SM, Yang B, Peng Y, Wang Y, Liu Z, Chen J, Qin J, Li R, Chin FY. A robust and accurate binning algorithm for metagenomic sequences with arbitrary species abundance ratio. Bioinformatics. 2011;27(11):1489-95.

32. Karlin S. Detecting anomalous gene clusters and pathogenicity islands in diverse bacterial genomes. Trends Microbiol. 2001:9(7):335-43.

33. Merkl R. SIGl: score-based identification of genomic islands. BMC Bioinformatics. 2004:5:22

34. Dufraigne C, Fertil B, Lespinats S, Giron A, Deschavanne P. Detection and characterization of horizontal transfers in prokaryotes using genomic signature. Nucleic Acids Res. 2005;33(1):e6.

35. Tsirigos A, Rigoutsos I. A new computational method for the detection of horizontal gene transfer events. Nucleic Acids Res. 2005;33(3):922-33. 
36. Blaisdell BE, Campbell AM, Karlin S. Similarities and dissimilarities of phage genomes. Proc Natl Acad Sci U S A. 1996;93(12):5854-9.

37. Robins $\mathrm{H}$, Krasnitz $\mathrm{M}$, Barak $\mathrm{H}$, Levine AJ. A relative-entropy algorithm for genomic fingerprinting captures host-phage similarities. J Bacteriol. 2005; 187(24):8370-4.

38. Pride DT, Wassenaar TM, Ghose C, Blaser MJ. Evidence of host-virus coevolution in tetranucleotide usage patterns of bacteriophages and eukaryotic viruses. BMC Genomics. 2006;7:8.

39. Mrazek J, Karlin S. Distinctive features of large complex virus genomes and proteomes. Proc Natl Acad Sci U S A. 2007;104(12):5127-32

40. Campbell A, Mrazek J, Karlin S. Genome signature comparisons among prokaryote, plasmid, and mitochondrial DNA. Proc Natl Acad Sci U S A. 1999;96(16):9184-9.

41. Suzuki H, Sota M, Brown CJ, Top EM. Using Mahalanobis distance to compare genomic signatures between bacterial plasmids and chromosomes. Nucleic Acids Res. 2008;36(22):e147.

42. Bohlin J, Skjerve E, Ussery DW. Reliability and applications of statistical methods based on oligonucleotide frequencies in bacterial and archaeal genomes. BMC Genomics. 2008;9:104.

43. Iverson VS, Morris RM, Frazar CD, Berthiaume CT, Morales R, Armbrust EV. Untangling genomes from metagenomes: revealing an uncultured class of marine Euryarchaeota. Science. 2012;335(6068):587-90.

44. Tyson GW, Lo I, Baker BJ, Allen EE, Hugenholtz P, Banfield JF. Genomedirected isolation of the key nitrogen fixer Leptospirillum ferrodiazotrophum sp. nov. from an acidophilic microbial community. Appl Environ Microbiol. 2005;71(10):6319-24

45. Mende DR, Sunagawa S, Zeller G, Bork P. Accurate and universal delineation of prokaryotic species. Nat Methods. 2013;10(9):881-4.

46. Kim M, Oh HS, Park SC, Chun J. Towards a taxonomic coherence between average nucleotide identity and 16S rRNA gene sequence similarity for species demarcation of prokaryotes. Int J Syst Evol Microbiol. 2014;64(Pt 2):346-51.

47. Karlin S, Ladunga I. Comparisons of eukaryotic genomic sequences. Proc Natl Acad Sci U S A. 1994:91(26):12832-6.

48. Karlin S. Global dinucleotide signatures and analysis of genomic heterogeneity. Curr Opin Microbiol. 1998;1(5):598-610.

49. Verger JM, Grimont F, Grimont PAD, Grayon M. Brucella, a monospecific genus as shown by deoxyribonucleic-acid hybridization. Int I Syst Bacteriol. 1985;35(3):292-5.

50. Verger JM, Grimont F, Grimont PAD, Grayon M. Taxonomy of the genus Brucella. Ann Inst Pasteur Mic. 1987;138(2):235-8.

51. Sankarasubramanian J, Vishnu US, Gunasekaran P, Rajendhran J. A genomewide SNP-based phylogenetic analysis distinguishes different biovars of Brucella suis. Infect Genet Evol. 2016;41:213-7.

52. Wattam AR, Foster JT, Mane SP, Beckstrom-Sternberg SM, BeckstromSternberg JM, Dickerman AW, Keim P, Pearson T, Shukla M, Ward DV, et al. Comparative phylogenomics and evolution of the Brucellae reveal a path to virulence. J Bacteriol. 2014;196(5):920-30.

53. Gibson JR, Owen RJ. Campylobacter infections : species identification and typing. Methods Mol Med. 1998;15:407-18.

54. Islam Z, Gilbert M, Mohammad QD, Klaij K, Li J, van Riis W, Tio-Gillen AP, Talukder KA, Willison HJ, van Belkum A, et al. Guillain-Barre syndromerelated campylobacter jejuni in Bangladesh: ganglioside mimicry and crossreactive antibodies. PLoS One. 2012;7(8):e43976.

55. Heikema AP, Jacobs BC, Horst-Kreft D, Huizinga R, Kuijf ML, Endtz HP, Samsom $\mathrm{JN}$, van Wamel WJ. Siglec-7 specifically recognizes campylobacter jejuni strains associated with oculomotor weakness in Guillain-Barre syndrome and Miller fisher syndrome. Clin Microbiol Infect. 2013;19(2):E106-12.

56. Miller WG, Parker $C T$, Heath S, Lastovica AJ. Identification of genomic differences between Campylobacter jejuni subsp. jejuni and C. jejuni subsp. doylei at the nap locus leads to the development of a C. jejuni subspeciation multiplex PCR method. BMC Microbiol. 2007;7:11.

57. Ochman H, Lawrence JG, Groisman EA. Lateral gene transfer and the nature of bacterial innovation. Nature. 2000:405(6784):299-304

58. Langille MG, Hsiao WW, Brinkman FS. Detecting genomic islands using bioinformatics approaches. Nat Rev Microbiol. 2010;8(5):373-82.

59. Moran NA. Microbial minimalism: genome reduction in bacterial pathogens. Cell. 2002;108(5):583-6.

60. Tauch A, Schneiker S, Selbitschka W, Puhler A, van Overbeek LS, Smalla K, Thomas CM, Bailey MJ, Forney LJ, Weightman A, et al. The complete nucleotide sequence and environmental distribution of the cryptic, conjugative, broad-host-range plasmid pIPO2 isolated from bacteria of the wheat rhizosphere. Microbiology. 2002;148(Pt 6):1637-53.

61. Takahashi Y, Shintani M, Takase N, Kazo Y, Kawamura F, Hara H, Nishida H, Okada K, Yamane H, Nojiri H. Modulation of primary cell function of host Pseudomonas bacteria by the conjugative plasmid pCAR1. Environ Microbiol. 2015;17(1):134-55.

62. Qi J, Luo H, Hao B. CVTree: a phylogenetic tree reconstruction tool based on whole genomes. Nucleic Acids Res. 2004;32(Web Server issue):W45-7.

63. Maiden MCJ, Bygraves JA, Feil EJ, Morelli G, Russell JE, Urwin R, Zhang Q, Zhou JL, Zurth K, Caugant DA. Multilocus sequence typing: a portable approach to the identification of clones within populations of pathogenic microorganisms. Proc Natl Acad Sci U S A. 1998;95(6):3140-5.

64. Zhou Y, Pope PB, Li S, Wen B, Tan F, Cheng S, Chen J, Yang J, Liu F, Lei X, et al. Omics-based interpretation of synergism in a soil-derived cellulosedegrading microbial community. Sci Rep. 2014;4:5288.

65. Vinh le V, Lang TV, Binh le T, Hoai TV. A two-phase binning algorithm using I-mer frequency on groups of non-overlapping reads. Algorithms Mol Biol. 2015;10(1):2.

66. Liao R, Zhang R, Guan J, Zhou S. A new unsupervised binning approach for metagenomic sequences based on $\mathrm{N}$-grams and automatic feature weighting. IEEE/ACM Trans Comput Biol Bioinform. 2014;11(1):42-54.

67. Kurtz S, Phillippy A, Delcher AL, Smoot M, Shumway M, Antonescu C, Salzberg SL. Versatile and open software for comparing large genomes. Genome Biol. 2004;5(2):R12.

68. Konstantinidis KT, Tiedje JM. Genomic insights that advance the species definition for prokaryotes. Proc Natl Acad Sci U S A. 2005;102(7):2567-72.

69. Mrazek J, Karlin S. Strand compositional asymmetry in bacterial and large viral genomes. Proc Natl Acad Sci U S A. 1998;95(7):3720-5.

70. Schbath S, Prum B, de Turckheim E. Exceptional motifs in different Markov chain models for a statistical analysis of DNA sequences. J Comput Biol. 1995;2(3):417-37.

\section{Publisher's Note}

Springer Nature remains neutral with regard to jurisdictional claims in published maps and institutional affiliations.

Ready to submit your research? Choose BMC and benefit from:

- fast, convenient online submission

- thorough peer review by experienced researchers in your field

- rapid publication on acceptance

- support for research data, including large and complex data types

- gold Open Access which fosters wider collaboration and increased citations

- maximum visibility for your research: over $100 \mathrm{M}$ website views per year

At BMC, research is always in progress.

Learn more biomedcentral.com/submissions 